\title{
Bariatric surgery: a potential cure for asthma?
}

\author{
Toufic A. Chaaban
}

Affiliation: Ohio State University, Wexner Medical Center, Columbus, OH, USA.

Correspondence: Toufic A. Chaaban, Ohio State University, Wexner Medical Center, 1793 Globe St, Columbus, $\mathrm{OH}$ 43212, USA. E-mail: t-chaabanahotmail.com

@ERSpublications

Asthma incidence and severity increase with obesity. There are possible positive effects of bariatric surgery on asthma control, exacerbation risk and medication use; however, data are scarce and not uniform, and larger well-designed trials are needed. http://bit.ly/2QQYIaE

Cite this article as: Chaaban TA. Bariatric surgery: a potential cure for asthma? Eur Respir Rev 2019; 28 : 190003 [https://doi.org/10.1183/16000617.0003-2019].

ABSTRACT Asthma incidence and severity are increased in obese populations. Systematic reviews have shown benefit from weight-loss interventions on asthma outcomes, but the role of bariatric surgery is still unclear. In this review, cohorts of obese asthmatic patients undergoing bariatric surgery were examined regarding different asthma outcomes. The available data on patients who were followed up showed improvements in asthma control, exacerbation risk, asthma-related hospitalisation, medication use and airway hyperresponsiveness, with some patients not requiring further treatment for asthma. Follow-up duration was variable, being mostly of 1 year, with some studies reporting long-term outcomes after 5 years. The studies reviewed had many limitations, including small numbers of patients, lack of control arm in some studies and lack of standardisation of asthma diagnosis, classification and outcome measures, in addition to possible reporting bias. Data on small numbers of patients also show the possibility of benefit exclusively in nonallergic asthma. Larger, more stringent clinical trials are needed before recommending bariatric surgery for treatment of asthma.

\section{Introduction}

Multiple epidemiological studies have shown increased incidence and prevalence of asthma in obese populations $[1,2]$. While confounding factors including comorbidities, smoking status and diet play a role, the association between obesity and asthma has multiple supportive arguments, including a dose-dependent relationship between body mass index (BMI) and asthma incidence [1], increased severity of asthma in obese patients [3,4], decreased response to treatment [5], worse asthma-related quality of life [3,4] and increased hospitalisations [4]. The pathophysiology of the asthma-obesity link is complex and involves mechanical [6], cytokine-mediated $[6,7]$ and genetic factors $[6,8]$, with complex genetic-environmental interactions [6]. Furthermore, weight-loss interventions have shown improvement in asthma outcomes [9-11]. In a recent meta-analysis of randomised clinical trials (RCTs) of weight-loss interventions, ОкоNIEwsкi et al. [11] reviewed four RCTs in children and six in adults of weight-loss interventions in obese asthmatics and concluded that, while the available studies are not uniform, weight loss leads to improvement in asthma-related quality of life, asthma control and lung function. They found no RCT evaluating the role of surgical interventions. This review will focus on the available data on the outcomes of asthma after bariatric surgery, including asthma symptoms and control, medication use, exacerbations and hospitalisations.

Provenance: Submitted article, peer reviewed.

Received: Jan 142019 | Accepted after revision: June 042019

Copyright $\odot$ ERS 2019. This article is open access and distributed under the terms of the Creative Commons Attribution Non-Commercial Licence 4.0. 


\section{Bariatric surgery and lung function}

Cohort studies have shown improvement of lung function in obese patients after bariatric surgery, mostly improvement in obesity-related restriction [12-17]. NGUYEN et al. [13] prospectively followed 104 obese patients after laparoscopic gastric surgery and showed that forced expiratory volume in $1 \mathrm{~s}$ (FEV1) improved by $12 \%$, forced vital capacity (FVC) improved by $9 \%$, peak expiratory flow improved by $15 \%$ and forced expiratory volume at $25-75 \%$ of FVC improved by $30 \%$ at 1 year. They also noted that improvement occurred as early as 3 months post intervention. Interestingly, the percentage of patients with an abnormal FEV1/FVC ratio (defined in their study as <0.8) decreased from $9.6 \%$ pre-operatively to $1.9 \%$ post-operatively $(\mathrm{p}=0.03)$. Similar results were shown by DÁvILA-CERVANTES et al. [14], who followed 30 patients without respiratory symptoms at baseline after vertical band gastroplasty with $53 \%$ excess weight loss. In addition to improvement in $\mathrm{FEV}_{1}, \mathrm{FVC}$, and inspiratory and expiratory force, none of the patients who had obstructive $(n=4)$ or restrictive $(n=4)$ disorders showed abnormalities 1 year after surgery.

Few studies have evaluated the effects of bariatric surgery on spirometry parameters in asthma [18-22]. BOULET et al. [18] reported improvement in FEV1, FVC, expiratory reserve volume, functional residual capacity (FRC) and FRC/total lung capacity (TLC) in 12 asthmatic patients 1 year after surgery; none of these patients had obstruction at baseline (defined as $\mathrm{FEV} 1 / \mathrm{FVC}<70 \%$ ) and only two patients had FEV1 $<80 \%$ of predicted value at baseline. In another longitudinal study [19], patients were divided into three groups: asthmatics and nonasthmatics who had bariatric surgery and obese asthmatics as control. FEV1, FRC and TLC improved in both groups after bariatric surgery, but FEV1/FVC, residual volume (RV) and RV/TLC did not change in the asthmatic group, suggesting that improvement is related to the restrictive component [19]. Al-ALWAN et al. [21], in their more rigorous study that compared obese females with nonallergic asthma to nonasthmatic controls, showed that in nonasthmatics the restrictive component improved while in asthmatics only FEV1 improved. They also reported differences in respiratory system resistance response, suggesting more effect on distal collapsible lung in asthmatic patients.

Taken together, these studies suggest positive effects on lung restriction of bariatric surgery in the general obese population and in patients with asthma, but the effect on airway obstruction is variable and might not translate into better asthma outcomes. The effect on bronchial responsiveness and airway inflammation, which is more relevant in asthma, will be discussed later in this review.

\section{Bariatric surgery and asthma control}

Asthma control can be assessed by validated scores and questionnaires, such as the Asthma Control Test (ACT) and Asthma Control Questionnaire (ACQ), and is a more clinically relevant outcome than spirometry for the asthmatic population. BOULET et al. [18] showed a significant decrease in ACQ score from a mean $\pm S D$ (range) of $5.6 \pm 5.1(0-17)$ to $0.3 \pm 0.5(0-1)(\mathrm{p}<0.03) 1$ year after bariatric surgery in 12 obese asthmatics, while the ACQ score remained unchanged in the control group. All six components of the score showed improvement. Improvement in ACT scores is also reported in other case series [23, 24]. MANisCALCo et al. [24] showed that ACT score significantly improved from 18.7 to 22.2 after surgery in 12 patients with intermittent or mild-to-moderate asthma and who lost at least $20 \%$ of their BMI. Improvement was seen in all five components of the score, whereas the ACT score was unchanged in the control group who had no change in their weight. Similar improvements in ACT and Mini Asthma Quality of Life Questionnaire (mini-AQLQ) were reported in another study and persisted after 5 years [22]. O'BRIEn et al. [25] used the Asthma Severity Score to assess 33 asthmatic patients before and after laparoscopic placement of an adjustable gastric band and found that the score decreased from 44.5 before operation to 14.3 at follow-up $(\mathrm{p}<0.001)$. 11 asthmatic patients were regarded as nonasthmatic on follow-up, having no clinical asthma and not requiring treatment. It is of note that all patients were evaluated by a respiratory physician pre-operatively, which makes the correct diagnosis of asthma more probable but makes better medical care a confounding factor. Consistent improvement in other questionnaire scores was also reported by Dixon et al. [26], where 10 patients were classified as having severe asthma pre-operatively and none of them retained this classification post-operatively, and by SIMARD et al. [27], who reported improvement in $79 \%$ of 17 asthmatic patients 2 years after bariatric surgery. One might argue that improvement might be related to improvements in other comorbidities, such as gastro-oesophageal reflux disease (GORD) or dyspnoea related to restriction from obesity, or to better general medical care for patients who had surgery, with more stringent follow-up, but the consistent benefit in all components of the questionnaires, including rescue medication use, night-time awakenings and wheezes, in addition to the presence and prospective follow-up of control groups in some series, signal better control of asthma as a disease.

\section{Bariatric surgery and asthma exacerbation}

Exacerbations play a significant role in the morbidity and mortality of asthma, leading to more emergency department visits and hospitalisations; thus, preventing exacerbations is a marker of asthma control and an 
important outcome in asthma therapeutic trials. Few case series have reported the effect of bariatric surgery on asthma exacerbations [23, 25, 28]. ToH et al. [23] reported three cases of severe treatment-resistant asthma on oral steroids who underwent bariatric surgery. All these patients had a significant decrease in emergency department asthma-related visits, from five, three and four visits in the year before surgery to one, zero and zero visits, respectively. O'BRIEN et al. [25] followed 709 obese patients closely after bariatric surgery, including 33 asthmatic patients. Nine patients reported one or more hospital admissions for acute asthma in the presurgical year; none of them was hospitalised for asthma following surgery. The regular follow-up by surgeons and general physicians mandated by the study protocol might have played a role. More insight comes from a well-designed study by HASEGAWA et al. [28], who reviewed administrative data from three states in the USA (California, Florida and Nebraska), including 2261 obese patients with asthma who underwent bariatric surgery, and compared the number of emergency department presentations for asthma as well as hospitalisations for acute asthma in four periods: 1324 months and 12 months pre-surgery, within 12 months after bariatric surgery and 13-24 months post-operatively. Patients served as their own self-controls. By including only patients who had emergency department visits or hospitalisation for asthma, this study focuses on patients with poorly controlled asthma. The results of this study showed significantly fewer emergency department visits or hospitalisations for asthma exacerbation within 12 months after bariatric surgery (risk 10.9\% (95\% CI 9.6$12.2 \%$ ), compared with $22.0 \%$ (95\% CI 20.3-23.7\%) in the reference period 13-24 months pre-surgery), corresponding to an odds ratio (OR) of 0.42 (95\% CI 0.35-0.50). Similarly, in the subsequent period of 13-24 months after bariatric surgery, the risk remained significantly lower (10.9\%, 95\% CI 9.6-12.2\%), corresponding to an OR of 0.42 (95\% CI 0.35-0.50). Notably, the benefit was seen in both outcomes (emergency department visits and hospitalisation). To exclude the effect of peri-operative care on asthma control, Hasegawa et al. [28] performed the same analysis on obese asthma patients who underwent nonbariatric surgery, and the risk of asthma-related emergency department admission and hospitalisation was not changed before and after operation.

\section{Bariatric surgery and asthma medication use}

Most case series have reported significant decrease in asthma medication use after bariatric surgery $[18,20,25,29-37]$ and, interestingly, they have also reported a proportion of patients stopping treatment completely, implying "curative" treatment for asthma. Before reaching any conclusion, it is important to determine whether the diagnosis of asthma is accurate in those patients, and examine the follow-up they received, how many patients were lost to follow-up and the baseline severity of their disease. MuRR et al. [37] reported that $100 \%$ of asthma patients in their cohort $(n=6)$ had marked decrease in medication use for asthma as assessed by follow-up questionnaires, with a mean \pm SEM follow-up of $30 \pm 2$ months, without further details on which medications were stopped or decreased and how "marked decrease" was defined. Dhabuwala et al. [30] followed 157 obese patients post-operatively after gastric bypass surgery; 34 of them had asthma diagnosed before the operation, requiring treatment. In 17 out of 34 patients, asthma was labelled as "resolved" (defined as not symptomatic and not requiring treatment), nine patients "improved" (defined as better control of the disease with the same or less medication use), five were unchanged and three had no follow-up. The severity of baseline asthma and the type of medications used was not reported in this manuscript. In the cohort described by O'Brien et al. [25] discussed earlier in this review, all 33 patients required less medication after the operation, 18 patients were requiring daily medication pre-operatively (suggesting that most patients had mild intermittent asthma), and only eight patients required daily treatment at post-operative follow-up. MACGREGOR et al. [31], in a retrospective study on 40 patients in 1993, presented more detailed data about the status of asthma in their patients. Patients were classified as severe if they had $>10$ exacerbations in a year and moderate if they had 6-10 exacerbations, which is different from the current definition. $70 \%$ were classified as severe and $30 \%$ as moderate; despite the frequency of exacerbations, only $72 \%$ of patients used daily medications at baseline and $27.5 \%$ were on as-needed medications only. Overall, $42 \%$ stopped medications for asthma and $24 \%$ remained on as-needed treatment. In the most severe group, 36\% stopped treatment completely, while $32 \%$ stayed on as-needed medications only. Although when evaluated by current understanding of asthma and treatment guidelines the treatment of these patients was inadequate given the absence of daily controller therapy in patients with frequent exacerbations, the importance of this study is in showing that patients with severe asthma might benefit from bariatric surgery. Completely stopping treatment for asthma after bariatric surgery was also reported in $66.7 \%$ [36] and $81 \%$ [20] of patients in other small series. More detailed description of medication use is reported in a retrospective cohort of 320 patients continuously enrolled in a large, southeast Michigan health maintenance organisation by SiKKA et al. [38]. Total respiratory medication prescription fills decreased by $49 \%$ (from 314 to 154 prescriptions) in the post-surgical year, with only $43.1 \%$ of patients filling prescriptions in the year before surgery also filling a prescription in the post-surgical surveillance period. 33 patients (10.4\%) were categorised as "stoppers" and six (1.9\%) as "starters" of respiratory medication. When the analysis was restricted to patients with 
physician-diagnosed asthma $(n=40)$, the results remained valid, with mean pre-surgical prescription fills of 7.0 decreasing to 3.8 in the post-surgical period. The analysis also showed significant decrease in $\beta_{2}$-agonists, inhaled steroids and oral steroids. Similarly, Lombardi et al. [34] reported a decrease in inhaled steroid usage in a prospective evaluation of 14 asthmatic patients after bariatric surgery, with a median decrease in the beclomethasone equivalent dose from $460 \mu \mathrm{g}$ at baseline to $218 \mu \mathrm{g} 1$ year after surgery.

\section{Bariatric surgery, airway hyperresponsiveness and asthma phenotype}

Airway hyperresponsiveness (AHR), measured by methacholine challenge tests, has been evaluated in multiple series of obese patients before and after bariatric surgery. BoulET et al. [18] showed that the mean provocative concentration causing a $20 \%$ fall in FEV1 (PC20) for methacholine improved from 0.84 to $6.2 \mathrm{mg} \cdot \mathrm{mL}^{-1}(\mathrm{p}<0.001)$ after 1 year in the surgery group $(\mathrm{n}=12)$, while it remained stable in the control asthma group who did not have bariatric surgery. Notably, change in AHR correlated with BMI change. van Huisstede et al. [19] also showed that, out of 24 patients who had a positive provocation test at baseline, 13 had negative tests on 12 months of follow-up after surgery.

The effect of bariatric surgery might differ according to asthma phenotype. While it is well known now that obesity is prevalent across all asthma phenotypes [39], including children [40], two distinct clusters can be identified: early-onset atopic asthma or allergic type, characterised by high T-helper cell type 2 (Th2) inflammation with improvement of symptoms with weight loss without disease remission [41-43]; and late-onset nonatopic asthma in obese females with little inflammation, sometimes neutrophilic inflammation [21, 41-44]. In late-onset nonatopic asthma, it is hypothesised that AHR and closure is caused by obesity, due to loss of outward tethering forces of the parenchyma on the airway, and disease remission is expected with significant weight loss [21, 41-44]. Dixon et al. [33] also showed that AHR improved significantly 12 months after bariatric surgery and, interestingly, when patients were stratified according to IgE levels, only patients with low IgE retained this benefit. CHAPMAN et al. [45], with some of the same co-authors, also analysed these patients in another report where they classified obese asthmatic patients as Th2-low $(n=8)$ with low IgE and late onset, and Th2-high $(n=5)$ with high IgE and early onset, and concluded that weight loss reduced sensitivity to airway closure in Th2-low but not Th2-high obese asthmatics. This might suggest that obese nonatopic patients with late onset might derive more benefit.

Bronchial biopsies were evaluated before and 1 year after surgery by van HuISSTEDE et al. [19] in 22 patients undergoing bariatric surgery. Eight of them had asthma. Submucosal cell counts of eosinophils, neutrophils, B-cells, macrophages, $\mathrm{CD} 4^{+} \mathrm{T}$-cells or $\mathrm{CD}^{+} \mathrm{T}$-cells did not change at 12 -month follow-up in either the asthma or nonasthma group; in contrast, mast cells decreased significantly at 12-month follow-up in the asthma group. An ongoing study at Duke University (Durham, NC, USA; ClinicalTrials. gov identifier NCT03115632) is currently recruiting patients to test the effect of bariatric surgery on airway fibrosis (measured on submucosal biopsies) in both asthmatics and nonasthmatics.

The effect of bariatric surgery on exhaled nitric oxide fraction, when evaluated, was variable, with significant decrease in asthmatics in one series [34] and no change in another [45].

\section{Bariatric surgery, inflammation and adipokines}

Obesity is known to induce a state of chronic inflammation, which has been implicated in the pathogenesis of associated diseases. Adipokines secreted by the adipose tissue play an important role in this association: leptin is known as a pro-inflammatory adipokine and is increased in the obese population, while adiponectin $[46,47]$ is an anti-inflammatory adipokine and is decreased $[39,48]$. In addition, obese patients have higher levels of pro-inflammatory mediators, including C-reactive protein, tumour necrosis factor (TNF)- $\alpha$ and inteleukin (IL)-6, among others. This imbalance has been implicated in the increased susceptibility of obese patients to AHR and bronchoconstriction. Airway epithelial cells express adiponectin and leptin receptors [47], and robust animal data support the role of increased leptin and decreased adiponectin in AHR [39, 49]. Human studies have also shown this trend, but results have not been consistent among all studies [50-53].

Bariatric surgery reverses this adipokine imbalance and it has been shown to lead to decreased leptin [54-57] and IL-6 [55-57] and increased adiponectin [55, 57]. This holds true in the obese asthma population. SIDELEVA et al. [58] studied adiponectin, leptin and other inflammatory markers in visceral fat biopsies, airway biopsies, bronchoalveolar lavage (BAL) and sera from obese asthmatic and nonasthmatic patients undergoing bariatric surgery. They showed decreased adiponectin and increased leptin in BAL from asthmatic patients, in addition to increased visceral fat leptin expression. These abnormalities were reversed after bariatric surgery but did not correlate with markers of airway inflammation, leading to the conclusion that these adipokines might have direct effects on the airway, independent of inflammation. Recently, BALTIER et al. [59] followed 19 obese asthma patients after bariatric surgery and showed significant decrease in systemic inflammatory markers and a decrease in sputum TNF- $\alpha$. 


\section{Bariatric surgery and asthma: possible mechanisms}

The mechanisms explaining the improvement and sometimes remission of asthma after bariatric surgery and resultant weight loss are probably multifactorial. Mechanical factors from significant weight loss play a significant role, as it has been shown that obese patients have tidal breathing at low volumes with a decrease in FRC and expiratory reserve volumes, which leads to collapse of the airways and hyperinflation [47]. Weight loss can reverse these abnormalities and leads to improvement in airway elastance [19, 21, 44]. As discussed, restoration of normal adipokine balance, with adiponectin increase and leptin decrease, might also play a role. Finally, remission of comorbidities known to complicate severe and difficult-to-treat asthma, such as obstructive sleep apnoea and GORD, contributes to disease control. Whether bariatric surgery improves response to inhaled corticosteroids or changes the delivery of inhaled medications to the distal airways is yet to be elucidated.

\section{Conclusion}

The available data, although not uniform, suggest that some obese patients with asthma may benefit from bariatric surgery with regard to control of the disease, exacerbation risk, medication use and quality of life. While obese asthma patients can be encouraged to undergo bariatric surgery if they have other indications, the available evidence is not enough to recommend surgery for obese asthmatic patients in the absence of other indications. Larger trials are needed, with focus on accurate asthma diagnosis and disease severity classification according to the current guidelines with optimal treatment. Standardised outcome measures and assessments are also needed to derive conclusions. In addition, it remains unclear which subgroup of patients, according to asthma phenotype or baseline BMI, will benefit from such therapy and whether the benefit is only related to the magnitude of weight loss or other hormonal and cytokine-related factors might play a role.

Conflict of interest: None declared.

\section{References}

1 Beuther DA, Sutherland ER. Overweight, obesity, and incident asthma: a meta-analysis of prospective epidemiologic studies. Am J Respir Crit Care Med 2007; 175: 661-666.

2 Camargo CA Jr, Weiss ST, Zhang S, et al. Prospective study of body mass index, weight change, and risk of adult-onset asthma in women. Arch Intern Med 1999; 159: 2582-2588.

3 Vortmann M, Eisner MD. BMI and health status among adults with asthma. Obesity 2008; 16: 146-152.

4 Mosen DM, Schatz M, Magid DJ, et al. The relationship between obesity and asthma severity and control in adults. J Allergy Clin Immunol 2008; 122: 507-511.

5 Boulet LP, Franssen E. Influence of obesity on response to fluticasone with or without salmeterol in moderate asthma. Respir Med 2007; 101: 2240-2247.

6 Tantisira KG, Weiss ST. Complex interactions in complex traits: obesity and asthma. Thorax 2001; 56: Suppl. 2, ii64-ii73.

7 Dandona P, Ghanim H, Monte SV, et al. Increase in the mediators of asthma in obesity and obesity with type 2 diabetes: reduction with weight loss. Obesity 2014; 22: 356-362.

8 Hallstrand TS, Fischer ME, Wurfel MM, et al. Genetic pleiotropy between asthma and obesity in a community-based sample of twins. J Allergy Clin Immunol 2005; 116: 1235-1241.

9 Adeniyi FB, Young T. Weight loss interventions for chronic asthma. Cochrane Database Syst Rev 2012; 7: CD009339.

10 Lv N, Xiao L, Ma J. Weight management interventions in adult and pediatric asthma populations: a systematic review. J Pulm Respir Med 2015; 5: 1000232.

11 Okoniewski W, Lu KD, Forno E. Weight loss for children and adults with obesity and asthma. A systematic review of randomized controlled trials. Ann Am Thorac Soc 2019; 16: 613-625.

12 Hewitt S, Humerfelt S, Søvik TT, et al. Long-term improvements in pulmonary function 5 years after bariatric surgery. Obes Surg 2014; 24: 705-711.

13 Nguyen NT, Hinojosa MW, Smith BR, et al. Improvement of restrictive and obstructive pulmonary mechanics following laparoscopic bariatric surgery. Surg Endosc 2009; 23: 808-812.

14 Dávila-Cervantes A, Domínguez-Cherit G, Borunda D, et al. Impact of surgically-induced weight loss on respiratory function: a prospective analysis. Obes Surg 2004; 14: 1389-1392.

15 Santana AN, Souza R, Martins AP, et al. The effect of massive weight loss on pulmonary function of morbid obese patients. Respir Med 2006; 100: 1100-1104.

16 de Souza SA, Faintuch J, Cecconello I. Spirometric function improves in the morbidly obese after 1-year post-surgery. Obes Surg 2010; 20: 1273-1277.

17 Olsén MF, Wiklund $\mathrm{M}$, Lönroth $\mathrm{H}$, et al. Respiratory function in superobese patients before and after bariatric surgery - a randomised controlled trial. Open Obes J 2012; 4: 28-34.

18 Boulet LP, Turcotte H, Martin J, et al. Effect of bariatric surgery on airway response and lung function in obese subjects with asthma. Respir Med 2012; 106: 651-660.

19 van Huisstede A, Rudolphus A, Castro Cabezas M, et al. Effect of bariatric surgery on asthma control, lung function and bronchial and systemic inflammation in morbidly obese subjects with asthma. Thorax 2015; 70: 659-667.

20 Spivak H, Hewitt MF, Onn A, et al. Weight loss and improvement of obesity-related illness in 500 U.S. patients following laparoscopic adjustable gastric banding procedure. Am J Surg 2005; 189: 27-32.

21 Al-Alwan A, Bates JH, Chapman DG, et al. The nonallergic asthma of obesity. A matter of distal lung compliance. Am J Respir Crit Care Med 2014; 189: 1494-1502. 
22 Maniscalco M, Zamparelli AS, Vitale DF, et al. Long-term effect of weight loss induced by bariatric surgery on asthma control and health related quality of life in asthmatic patients with severe obesity: a pilot study. Respir Med 2017; 130: 69-74.

23 Toh JJ, Pasupathy S, Poopalalingam RA, et al. Can bariatric surgery be performed safely in patients with severe treatment-resistant asthma? Obes Surg 2014; 24: 334-336.

24 Maniscalco M, Zedda A, Faraone S, et al. Weight loss and asthma control in severely obese asthmatic females. Respir Med 2008; 102: 102-108.

25 O'Brien PE, Dixon JB, Brown W, et al. The laparoscopic adjustable gastric band (Lap-Band): a prospective study of medium-term effects on weight, health and quality of life. Obes Surg 2002; 12: 652-660.

26 Dixon JB, Chapman L, O’Brien P. Marked improvement in asthma after Lap-Band surgery for morbid obesity. Obes Surg 1999; 9: 385-389.

27 Simard B, Turcotte H, Marceau P, et al. Asthma and sleep apnea in patients with morbid obesity: outcome after bariatric surgery. Obes Surg 2004; 14: 1381-1388.

28 Hasegawa K, Tsugawa Y, Chang Y, et al. Risk of an asthma exacerbation after bariatric surgery in adults. J Allergy Clin Immunol 2015; 136: 288-294.

29 Brancatisano A, Wahlroos S, Brancatisano R. Improvement in comorbid illness after placement of the Swedish Adjustable Gastric Band. Surg Obes Relat Dis 2008; 4: Suppl. 3, S39-S46.

30 Dhabuwala A, Cannan RJ, Stubbs RS. Improvement in co-morbidities following weight loss from gastric bypass surgery. Obes Surg 2000; 10: 428-435.

31 Macgregor AM, Greenberg RA. Effect of surgically induced weight loss on asthma in the morbidly obese. Obes Surg 1993; 3: 15-21.

32 Hall JC, Watts JM, O’Brien PE, et al. Gastric surgery for morbid obesity. The Adelaide Study. Ann Surg 1990; 211: 419-427.

33 Dixon AE, Pratley RE, Forgione PM, et al. Effects of obesity and bariatric surgery on airway hyperresponsiveness, asthma control, and inflammation. J Allergy Clin Immunol 2011; 128: 508-515.

34 Lombardi C, Gargioni S, Gardinazzi A, et al. Impact of bariatric surgery on pulmonary function and nitric oxide in asthmatic and non-asthmatic obese patients. J Asthma 2011; 48: 553-557.

35 Ahroni JH, Montgomery KF, Watkins BM. Laparoscopic adjustable gastric banding: weight loss, co-morbidities, medication usage and quality of life at one year. Obes Surg 2005; 15: 641-647.

36 Sultan S, Parikh M, Youn H, et al. Early U.S. outcomes after laparoscopic adjustable gastric banding in patients with a body mass index less than $35 \mathrm{~kg} / \mathrm{m}^{2}$. Surg Endosc 2009; 23: 1569-1573.

37 Murr MM, Siadati MR, Sarr MG. Results of bariatric surgery for morbid obesity in patients older than 50 years. Obes Surg 1995; 5: 399-402.

38 Sikka N, Wegienka G, Havstad S, et al. Respiratory medication prescriptions before and after bariatric surgery. Ann Allergy Asthma Immunol 2010; 104: 326-330.

39 Sood A, Shore SA. Adiponectin, leptin, and resistin in asthma: basic mechanisms through population studies. J Allergy 2013; 2013: 785835.

40 Lang JE, Hossain J, Dixon AE, et al. Does age impact the obese asthma phenotype? Longitudinal asthma control, airway function, and airflow perception among mild persistent asthmatics. Chest 2011; 140: 1524-1533.

41 Bates JH, Poynter ME, Frodella CM, et al. Pathophysiology to phenotype in the asthma of obesity. Ann Am Thorac Soc 2017; 14: Suppl. 5, S395-S398.

42 Bates JH. Physiological mechanisms of airway hyperresponsiveness in obese asthma. Am J Respir Cell Mol Biol 2016; 54: 618-623.

43 Peters U, Dixon AE, Forno E. Obesity and asthma. J Allergy Clin Immunol 2018; 141: 1169-1179.

44 Dixon AE, Poynter ME. Mechanisms of asthma in obesity. Pleiotropic aspects of obesity produce distinct asthma phenotypes. Am J Respir Cell Mol Biol 2016; 54: 601-608.

45 Chapman DG, Irvin CG, Kaminsky DA, et al. Influence of distinct asthma phenotypes on lung function following weight loss in the obese. Respirology 2014; 19: 1170-1177.

46 Desai D, Newby C, Symon FA, et al. Elevated sputum interleukin-5 and submucosal eosinophilia in obese individuals with severe asthma. Am J Respir Crit Care Med 2013; 188: 657-663.

47 Baffi CW, Winnica DE, Holguin F. Asthma and obesity: mechanisms and clinical implications. Asthma Res Pract 2015; 1: 1 .

48 Ulrik CS. Asthma and obesity: is weight reduction the key to achieve asthma control? Curr Opin Pulm Med 2016; 22: 69-73.

49 Leiria LO, Martins MA, Saad MJ. Obesity and asthma: beyond Th2 inflammation. Metabolism 2015; 64: 172-181.

50 Sutherland TJ, Sears MR, McLachlan CR, et al. Leptin, adiponectin, and asthma: findings from a population-based cohort study. Ann Allergy Asthma Immunol 2009; 103: 101-107.

51 Ali Assad N, Sood A. Leptin, adiponectin and pulmonary diseases. Biochimie 2012; 94: 2180-2189.

52 Newson RB, Jones M, Forsberg B, et al. The association of asthma, nasal allergies, and positive skin prick tests with obesity, leptin, and adiponectin. Clin Exp Allergy 2014; 44: 250-260.

53 Holguin F, Rojas M, Brown LA, et al. Airway and plasma leptin and adiponectin in lean and obese asthmatics and controls. J Asthma 2011; 48: 217-223.

54 Kalinowski P, Paluszkiewicz R, Wróblewski T, et al. Ghrelin, leptin, and glycemic control after sleeve gastrectomy versus Roux-en-Y gastric bypass - results of a randomized clinical trial. Surg Obes Relat Dis 2017; 13: 181-188.

55 Marantos G, Daskalakis M, Karkavitsas N, et al. Changes in metabolic profile and adipoinsular axis in morbidly obese premenopausal females treated with restrictive bariatric surgery. World J Surg 2011; 35: 2022-2030.

56 Felipo V, Urios A, García-Torres ML, et al. Alterations in adipocytokines and cGMP homeostasis in morbid obesity patients reverse after bariatric surgery. Obesity 2013; 21: 229-237.

57 Kelly AS, Ryder JR, Marlatt KL, et al. Changes in inflammation, oxidative stress and adipokines following bariatric surgery among adolescents with severe obesity. Int J Obes 2016; 40: 275-280.

58 Sideleva O, Suratt BT, Black KE, et al. Obesity and asthma: an inflammatory disease of adipose tissue not the airway. Am J Respir Crit Care Med 2012; 186: 598-605.

59 Baltieri L, Cazzo E, de Souza AL, et al. Influence of weight loss on pulmonary function and levels of adipokines among asthmatic individuals with obesity: one-year follow-up. Respir Med 2018; 145: 48-56. 